\title{
A characterization and prognosis prediction model for primary squamous cell carcinoma of the thyroid
}

\author{
Xingyu Zhang ${ }^{1 \#}$, Gangcai Zhu ${ }^{2 \#}$, Bin Tang ${ }^{1}$, Huimei Huang ${ }^{2}$, Changhan Chen ${ }^{1}$, Siyuan Zheng ${ }^{1}$, \\ Yuting Pu ${ }^{1}$, Yimin $\mathrm{Xu}^{1}$, Gang Wang ${ }^{1}$, Donghai Huang ${ }^{1,3,4,5}$, Yong Liu ${ }^{1,3,4}$, Xin Zhang ${ }^{1,3,4,5}$ \\ ${ }^{1}$ Department of Otolaryngology-Head and Neck Surgery, Xiangya Hospital, Central South University, Changsha, China; ${ }^{2}$ Department of \\ Otolaryngology-Head and Neck Surgery, The Second Xiangya Hospital, Central South University, Changsha, China; ${ }^{3}$ Otolaryngology Major \\ Disease Research Key Laboratory of Hunan Province, Changsha, China; ${ }^{4}$ Clinical Research Center for Pharyngolaryngeal Diseases and Voice \\ Disorders in Hunan Province, Changsha, China; ${ }^{5}$ National Clinical Research Center for Geriatric Disorders (Xiangya Hospital), Changsha, China \\ Contributions: (I) Conception and design: X Zhang, G Zhu, Y Liu, D Huang, X Zhang; (II) Administrative support: X Zhang; (III) Provision of study \\ materials or patients: G Zhu; (IV) Collection and assembly of data: X Zhang; (V) Data analysis and interpretation: X Zhang, G Zhu; (VI) Manuscript \\ writing: All authors; (VII) Final approval of manuscript: All authors. \\ \#These authors contributed equally to this work. \\ Correspondence to: Yong Liu, MD, PhD; Xin Zhang, MD, PhD. Department of Otolaryngology-Head and Neck Surgery, Xiangya Hospital, Central \\ South University, Changsha 410008, China. Email: liuyongent@csu.edu.cn; xinzhang@csu.edu.cn.
}

Backgroundk Primary squamous cell carcinoma of the thyroid (PSCCTh) is a sporadic malignancy arising from the thyroid gland. The factors that affect treatment and survival in patients with PSCCTh remain unclear. Our study aims to characterize PSCCTh and establish a prognosis prediction model for patients with PSCCTh.

Methods: Clinical data and follow-up information for 277 patients from 1973 to 2016 were collected from the Surveillance, Epidemiology, and End Results Program (SEER) 18-registry database (RRID:SCR_003293). Univariate and multivariate Cox proportional hazards analyses and nomogram modeling of potential prognostic factors were conducted.

Results: Among the collected patient cases, 57\% were female and $43 \%$ were male. The median survival of all cases was 6 months; by gender, median survival was 5 and 8 months in the female and male groups, respectively. Univariate and multivariate Cox proportional hazards analyses revealed that age, extent of disease (EOD), $\mathrm{T}$ stage, $\mathrm{N}$ stage, and treatment were independent prognostic indicators for overall survival (OS) and disease-specific survival (DSS) in patients with PSCCTh. In addition, it was confirmed that the established nomogram model had good consistency and discrimination for PSCCTh prognosis as measured by the concordance index (C-index), area under the receiver operating characteristic (ROC) curve (AUC), and calibration curves.

Conclusions: Our study indicates that age, EOD, T stage, N stage, and treatment may correlate with OS and DSS in patients with PSCCTh. Importantly, our nomogram prediction model, constructed using parameters including age, $\mathrm{T}$ stage, $\mathrm{N}$ stage, and treatment, may assist physicians in evaluating patients' prognoses and providing precise therapy for PSCCTh.

Keywords: Primary squamous cell carcinoma of the thyroid (PSCCTh); Surveillance, Epidemiology, and End Results Program database (SEER database); prognosis; nomogram; primary squamous cell carcinoma

Submitted Nov 23, 2020. Accepted for publication Feb 20, 2021.

doi: $10.21037 / \mathrm{gs}-20-847$

View this article at: http://dx.doi.org/10.21037/gs-20-847 


\section{Introduction}

Primary squamous cell carcinoma of the thyroid (PSCCTh), a rare malignancy accounting for less than $1 \%$ of all primary carcinomas of the thyroid gland (1), was first reported by Vonkarst et al. in 1858 (2). The most common symptom of PSCCTh, as with other thyroid cancers, including papillary carcinoma, is the development of a neck mass $(60 \%)$, followed by difficulty breathing or swallowing (20\%) and voice changes (15\%) (3). The primary cause of mortality in PSCCTh is the involvement of the respiratory system due to direct invasion or compression of the trachea (4). However, the prognosis of PSCCTh patients is much worse than for patients with other types of thyroid cancers. Papillary thyroid carcinoma (PTC) and follicular thyroid carcinoma (FTC) account for over $90 \%$ of all thyroid cancer cases (5). Both PTC and FTC have good prognoses and survival rates, with an average survival of over 48 months (6), 5-year overall survival (OS) rates of $87.0 \%$ (7), and 10-year OS rates of $76.8 \%$ (8). Anaplastic thyroid carcinoma (ATC) accounts for less than $2 \%$ of all thyroid tumors, and $14-39 \%$ of deaths related to thyroid carcinoma are attributable to ATC (9). The median survival of patients with ATC is approximately 3 months; only $10-15 \%$ of patients survive 2 years after symptom presentation $(10,11)$, and the 1 -year survival rate is only $19.1 \%$ (5). The clinical characteristics of PSCCTh resemble those of ATC, and its prognosis is similarly poor. The average survival time of patients with PSCCTh is approximately 6.5 months (12), and the 1-year survival rate is $22.7 \%$ (13).

The gold standard for the diagnosis of PSCCTh requires a complete pathological examination of surgical specimens, as it is difficult to distinguish a PSCCTh from one originating in other anatomical sites if it invades adjacent tissue (14). In addition, the source of thyroid squamous cells is still unknown. To date, three theories regarding the origin of thyroid squamous cells have emerged: (I) the embryonic nest theory proposes that squamous cells may be derived from embryonic remnants, such as the thyroglossal duct, thymic epithelium, and bronchial body (15); (II) the metaplastic theory puts forward the idea that environmental stimuli (such as inflammation and Hashimoto's thyroiditis) could lead to squamous metaplasia (16); and (III) the dedifferentiation theory posits that existing papillary, follicular, medullary, and undifferentiated thyroid cancers differentiate into squamous cell carcinoma (17). However, these hypotheses require further study to be verified.

Due to its atypical characteristics and low incidence, there are a limited number of studies in the literature contributing to our understanding of PSCCTh. Most available articles are either case reports or small case series from a single institution (18-20). To better understand PSCCTh, the research community desperately needs studies that enroll more patients across multiple hospitals. The Surveillance, Epidemiology, and End Results Program (SEER) 18-registry database collects nationwide clinicopathological data and basic follow-up information in the U.S., which makes it a fantastic tool for studying rare cancers (21). Cancer-related data in the database is standardized using SEERStat software and is regularly updated and released. Consequently, by encompassing a large patient cohort and presenting a powerful statistical utility, the SEER database may provide us a solid clinical reference for PSCCTh. To our knowledge, there are three PSCCTh studies based on the SEER registry and the National Cancer Database (NCDB, another nationwide oncology database) (22-24). These studies examined factors impacting the prognosis of PSCCTh, which laid the foundation for further research. However, there are still some unclarified points. For example, it remains undetermined whether there is a cutoff age that could help clinicians predict prognosis. Additionally, it is unclear whether there are differences in prognostic factors for PSCCTh patients who meet various criteria (e.g., metastasis/no metastasis, radiotherapy/no radiotherapy). To fill these gaps, we applied the SEER database to characterize and analyze clinical factors, such as age, presence of distant metastasis, and treatment regimen, that affect the prognosis of patients with PSCCTh. This study is the first to generate a nomogram prognosis prediction model for PSCCTh. We present the following article in accordance with the TRIPOD reporting checklist (available at http://dx.doi. org/10.21037/gs-20-847).

\section{Methods}

The study was conducted in accordance with the Declaration of Helsinki (as revised in 2013). Because the data in the SEER database contain no personal identifiers and are publicly available after permission is obtained, the Medical Ethics Committee of Xiangya Hospital, Central South University in Changsha, China exempted this study from review. 


\section{Data collection}

All PSCCTh patients enrolled in this study were more than 25 years of age and were enrolled from 1973 to 2016. Squamous cell carcinoma was identified according to the International Classification of Diseases for Oncology 3rd edition (ICD-O-3)/World Health Organization (WHO) 2008 histology and behavior codes from 8070/3 to 8076/3 and the thyroid primary site code C739. Primary data were extracted from each individual cancer case in the database. Demographic variables included the patient record number, age at diagnosis, years since diagnosis, and gender. Based on the AJCCT (American Joint Committee on Cancer Staging) 6th edition (2004+), the clinical factors of interest included length of survival; cause of death (COD) according to the site record; vital status recode; ICD-O-3 histologic type; tumor grade (well/moderately/poorly differentiated and undifferentiated); tumor size via North American Association of Central Cancer Registries (NAACCR) \#2800 (information on tumor size, available for 2004+), \#780 (available for 1988-2003), and \#860 (available for 1983-1987); extent of disease (EOD)-extent via NAACCR \#790 (information on the EOD, available for 1988-2003), \#860 (available for 1983-1987), and \#840 (available for 1975-1982); the history of surgical treatment with radiation or chemotherapy; T stage; $\mathrm{N}$ stage; and $M$ stage.

\section{Tumor case cobort}

A total of 277 patients were enrolled in the study, which included five distinct morphological subtypes within PSCCTh: 8070/3: squamous cell carcinoma, not otherwise specified (NOS); 8071/3: keratinizing; 8072/3: large cell, nonkeratinizing (LCN); 8074/3: spindle cell (SC); and 8076/3: micro-invasive (MI). Certain continuous variables were converted into dichotomous variables due to the limited sample size or asymmetrical distribution. With regard to age, we analyzed the impact of different ages on patient prognosis and selected 70 years old as the point at which to divide the population into two groups (Figure 1). We defined three groups based on treatment strategy: surgery with adjuvant radiation therapy, surgery with no radiation, and no surgical treatment. Tumor sizes ranged from 7 to $150 \mathrm{~mm}$, with a mean size of $51 \mathrm{~mm}$ and a median size of $49 \mathrm{~mm}$; therefore, $50 \mathrm{~mm}$ was selected as the size standard used to divide the tumors into two groups. According to the AJCCT 6th edition, T classification was grouped into early-stage (T1, T2, and T3) and advanced- stage (T4a, T4b, and T4NOS).

SEER EOD coding incorporates six digits, of which three represent the size and/or involvement of the primary tumor, two represent the tumor extension, and one digit is a general code for lymph node involvement. For the four additional digits, two numbers indicate the presence or absence of pathologically positive lymph nodes, and two numbers indicate the total number of lymph nodes examined. EOD codes based on clinical, operative, and pathological diagnoses provide comprehensive information on tumor development and metastasis (25). Following the SEER EOD 1988 Codes and Coding Instructions (3rd edition, 1998), enrolled patients were classified into two groups: those with metastasis (score $\geq 85$ ) and those without (score $<85$ ). Summary data for all the enrolled patients are presented in Table 1.

\section{Statistical analysis}

All analyses were conducted using SPSS software version 22.0 (IBM Corp., New York, RRID:SCR_019096). KaplanMeier survival analyses (log-rank test) and multivariate Cox proportional hazards analyses were performed to evaluate OS and disease-specific survival (DSS) based on potential prognostic factors, such as age at diagnosis, tumor grade, surgical treatment, EOD, and TNM stage. $\mathrm{P}<0.05$ was considered statistically significant. Following the univariate analyses, as some indicators correlated with each other, it was necessary to summarize and extract independent factors through comparative tests before conducting the multivariate Cox analyses (23). We subsequently excluded non-independent variables and variables that did not show statistical significance. The significant variables $(\mathrm{P}<0.1)$ identified in univariate analyses were included in the stepwise regression analyses (backward method), which ultimately determined the variables included in the Cox proportional hazards model (26).

\section{Construction and verification of the prediction model}

The nomogram survival model was established based on the Cox proportional hazards model and survival package of $\mathrm{R}$ software (27). The independent prognostic factors in multivariate Cox analyses were converted into regression coefficients using the software to determine the scores of the predictive factors, and a nomogram was drawn for the prediction model. Based on the patient's situation, we could draw vertical lines on the nomogram to calculate the scores 
A

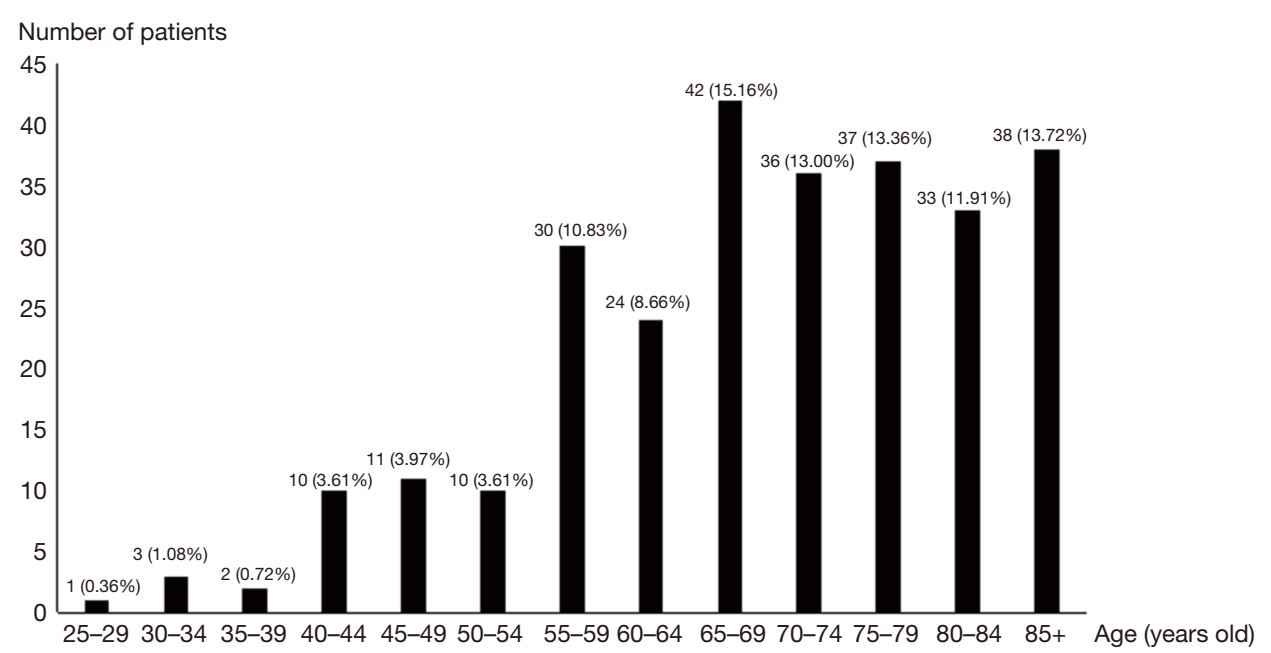

B

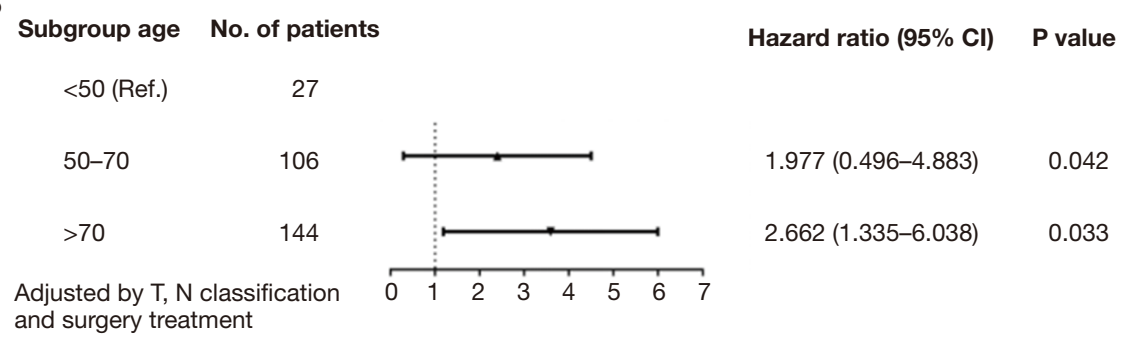

Figure 1 The age distribution with each 5-year age cohort in PSCCTh patients (A). Forest plot of multivariate analysis of each factor's ability in predicting OS (B). PSCCTh, primary squamous cell carcinoma of the thyroid; OS, overall survival; CI, confidence interval.

corresponding to specific patient factors and add the scores to obtain the total score. The vertical line corresponding to the total score intersects the lines representing the probability of survival at 1,3 , and 5 years to yield the predicted survival probability for a specific PSCCTh patient. The bootstrap self-sampling method was used to resample the verification group data 1,000 times to obtain a calibration curve that indicated the degree of consistency between the predicted survival rate and the actual survival rate while avoiding overfitting the model (28); good prediction consistency was established when scattered points fell on the $45^{\circ}$ diagonal reference line. The discrimination was measured via the area under the receiver operating characteristic (ROC) curve (AUC) and concordance index (C-index), resulting in values ranging from 0 to 1 . These values for our model were close to 1 , demonstrating that it had discrimination capacity.

\section{Results}

\section{Patient characteristics}

A total of 277 patients with PSCCTh were selected from the SEER database. As shown in Table 1, female and male patients accounted for $57 \%$ and $43 \%$ of the cohort, respectively. The median age of these patients was 72 (range, 25-89) years old. Most of the patients were 65-69 years old [hazard ratio (HR): $0.483(0.398-0.767)$, $\mathrm{P}=0.002]$, and $52 \%$ of patients in the primary cohort were 70 years old or older (Figure 1). The distribution of tumor grade was $7 \%, 21 \%, 50 \%$, and $22 \%$ for grades I, II, III, and IV, respectively. There were $28(10.1 \%)$ patients with T1-3 tumors and $85(30.7 \%)$ with T4 tumors. A total of $60(21.7 \%)$ patients had a distant metastasis. A total of $29.2 \%$ received surgery with radiotherapy, and $20.9 \%$ were treated with surgery only. 
Table 1 Demographic, clinical, and pathologic characteristics in 277 patients with primary squamous cell carcinoma in the thyroid, 1973-2016

\begin{tabular}{|c|c|}
\hline Characteristics & No. of patients (\%) \\
\hline Male & $119(43.0)$ \\
\hline Female & $158(57.0)$ \\
\hline \multicolumn{2}{|l|}{ Age (years old) } \\
\hline$\geq 70$ & $144(52.0)$ \\
\hline \multicolumn{2}{|l|}{ Race } \\
\hline White & $226(81.5)$ \\
\hline Blacks & $21(7.5)$ \\
\hline \multicolumn{2}{|l|}{ Insurance status } \\
\hline Insured & $116(41.9)$ \\
\hline Uninsured & $3(1.1)$ \\
\hline Unknown & $158(57.0)$ \\
\hline \multicolumn{2}{|l|}{ Marriage } \\
\hline Married & $148(53.4)$ \\
\hline Single (including divorced, widowed, and never married) & $110(39.7)$ \\
\hline Unknown & $19(6.9)$ \\
\hline Unknown & $83(30.0)$ \\
\hline \multicolumn{2}{|l|}{ Tumor size (mm) } \\
\hline$<50$ & $47(17.0)$ \\
\hline$\geq 50$ & $46(16.6)$ \\
\hline Unknown & $184(66.4)$ \\
\hline \multicolumn{2}{|l|}{ Subtype } \\
\hline 8070/3: squamous cell carcinoma, NOS & $240(86.6)$ \\
\hline 8071/3: squamous cell carcinoma, keratinizing, NOS & $20(7.3)$ \\
\hline 8072/3: squamous cell carcinoma, LCN & $3(1.1)$ \\
\hline 8074/3: squamous cell carcinoma, SC & $13(4.7)$ \\
\hline 8076/3: squamous cell carcinoma, MI & $1(0.3)$ \\
\hline
\end{tabular}

Table 1 (continued) 
Table 1 (continued)

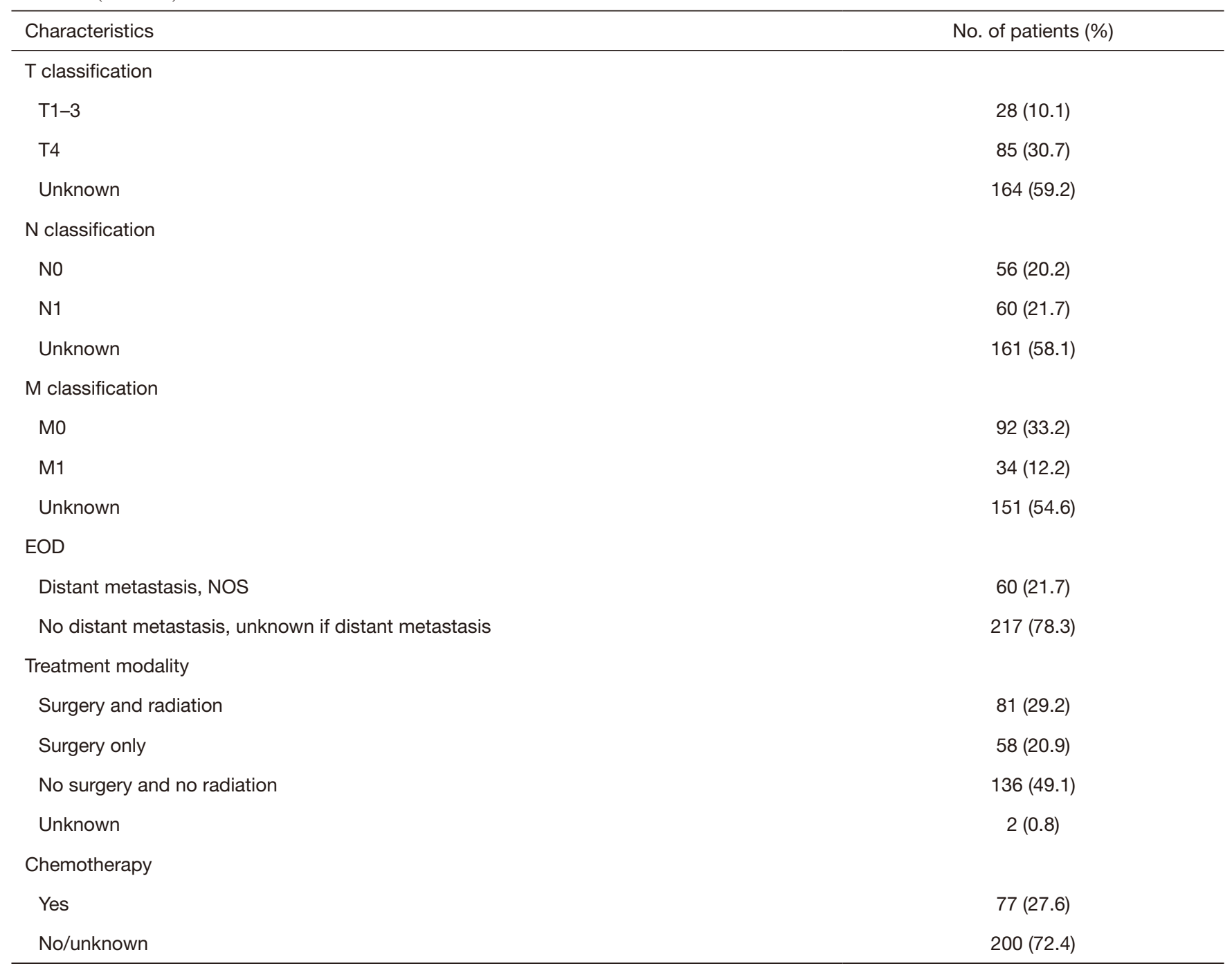

NOS, not otherwise specified; LCN, large cell, nonkeratinizing; SC, spindle cell; MI, micro-invasive; EOD, extent of disease.

\section{Survival outcomes}

Older patients (age $\geq 70$ ) constituted $76 \%$ of patients with a median survival time of 4 months, while younger patients (age $<70$ ) demonstrated a longer median survival time of 13 months. In the survival analyses, as shown in Table 2, OS and DSS were significantly associated with age at diagnosis (OS, $\mathrm{P}<0.001$; DSS, $\mathrm{P}=0.001$ ) but not correlated with sex (OS, $\mathrm{P}=0.524$; DSS, $\mathrm{P}=0.571$ ). The median survival of patients with tumor grades I, II, III, and IV was 24, 9, 4, and 3 months (OS, $\mathrm{P}=0.001$ ), but this factor did not have a significant impact on DSS $(\mathrm{P}=0.079)$. In this cohort, T4 patients had a median survival of 3 months, which was significantly shorter than the 19 months for T1-T3 patients (OS, $\mathrm{P}<0.001$; DSS, $\mathrm{P}=0.002$ ). The $\mathrm{N}$ stage showed statistical significance in the prediction of $\mathrm{OS}(\mathrm{P}=0.023)$ but had no significant impact on DSS $(\mathrm{P}=0.075)$. The median survival in patients with EOD was 5 months, while patients without EOD had a median survival time of 11 months. The Kaplan-Meier curves also showed that no EOD was associated with better OS or DSS (OS, $\mathrm{P}<0.001$; DSS, $\mathrm{P}=0.007)$. The median survival of patients who received surgical treatment was also significantly higher than that of patients who did not receive surgery (surgery with radiation: 7 months; surgery only: 6 months; no surgery: 
Table 2 Univariate analysis of prognostic factors for primary squamous cell carcinoma in thyroid

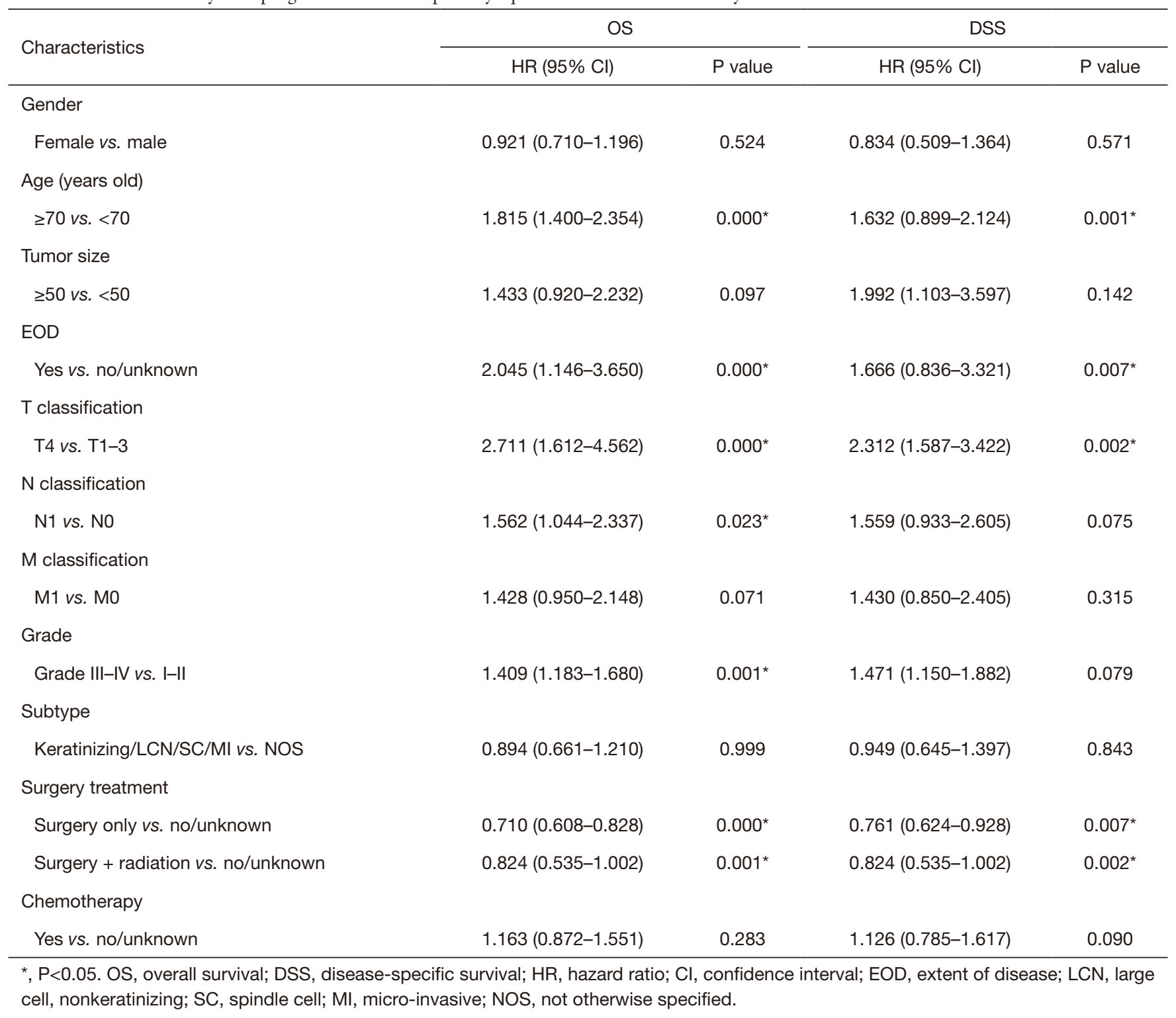

3 months), and those prognostic indicators were significantly different (OS, $\mathrm{P}<0.001$; DSS, $\mathrm{P}=0.002)$. As shown in Figure 2, indicators with statistical significance with respect to OS and DSS were used to draw the survival curves for OS.

\section{Multivariable Cox proportional hazards analyses}

Indicators with a $\mathrm{P}$ value $<0.1$ in the univariate analyses were further analyzed in multivariate analyses using a backward model selection procedure following comparative tests (as shown in Table S1), and significant variables $(\mathrm{P}<0.05)$ remained in the final Cox model. As shown in Table 3, age [OS, HR: 1.464, 95\% confidence interval (CI): (1.084-1.978), $\mathrm{P}=0.013$; DSS, HR: 2.238, 95\% CI: (1.076-4.655), $\mathrm{P}=0.031$ ], T stage [OS, HR: 2.684, 95\% CI: (1.553-4.639), P<0.001; DSS, HR: 4.500, $95 \%$ CI: (1.760-11.505), $\mathrm{P}=0.002], \mathrm{N}$ stage [OS, HR: 2.147, 95\% CI: (1.345-3.428), $\mathrm{P}=0.001$; DSS, HR: 1.853 , 95\% CI: (1.024-3.352), $\mathrm{P}=0.041]$, surgery [OS, HR: 0.587, 95\% CI: (0.254-0.869); DSS, HR: 0.405, 95\% CI: (0.1040.750)] and surgery + radiation [OS, HR: $0.603,95 \%$ CI: 
A

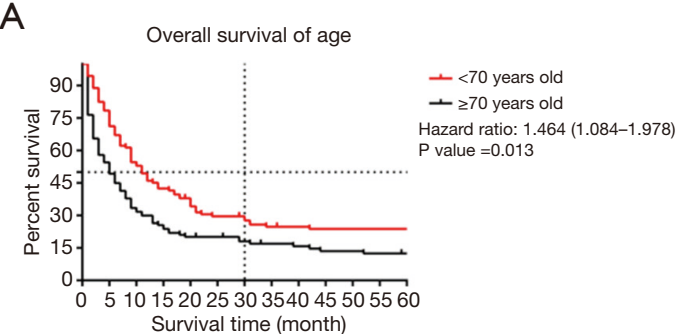

C

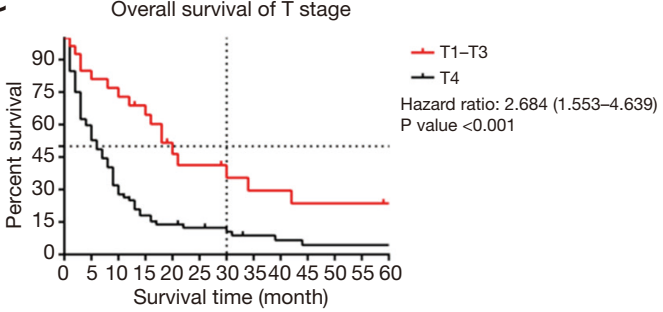

B

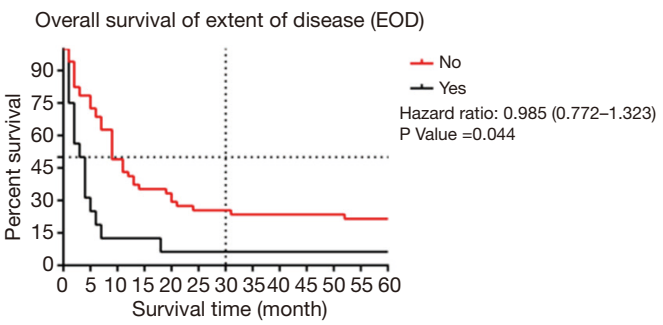

D

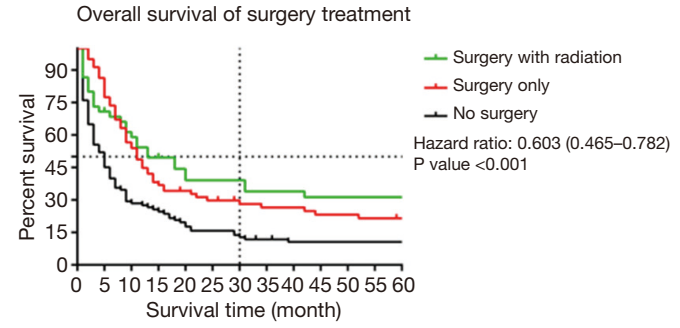

Figure 2 Kaplan-Meier curves of OS based on age (A), EOD (B), T classification (C), and surgery treatment (D). OS, overall survival; EOD, extent of disease.

Table 3 Multivariable Cox proportional hazards analyses for prognostic factors among clinical variables

\begin{tabular}{|c|c|c|c|c|}
\hline Characteristics & \multicolumn{2}{|l|}{ os } & \multicolumn{2}{|l|}{ DSS } \\
\hline \multicolumn{5}{|l|}{ Age (years old) } \\
\hline$\geq 70$ vs. $<70$ & $1.464(1.084-1.978)$ & $0.013^{*}$ & $2.238(1.076-4.655)$ & $0.031^{*}$ \\
\hline \multicolumn{5}{|l|}{ T classification } \\
\hline \multicolumn{5}{|l|}{$\mathrm{N}$ classification } \\
\hline N1 vs. N0 & $2.147(1.345-3.428)$ & $0.001^{*}$ & $1.853(1.024-3.352)$ & $0.041^{*}$ \\
\hline \multicolumn{5}{|l|}{ Surgery treatment } \\
\hline Surgery only vs. no/unknown & $0.587(0.254-0.869)$ & $0.001^{*}$ & $0.405(0.104-0.750)$ & $0.011^{*}$ \\
\hline
\end{tabular}

*, $\mathrm{P}<0.05$. OS, overall survival; DSS, disease-specific survival; $\mathrm{HR}$, hazard ratio; $\mathrm{Cl}$, confidence interval.

(0.465-0.782); DSS, HR: 0.662, 95\% CI: (0.479-0.915)] were independent prognostic factors in patients with PSCCTh.

\section{Stratified subgroup analyses}

According to the 2020 NCCN guidelines (https://www. nccn.org), age is a crucial initial indicator of the clinical stage in patients with PTC, which may skew the survival outcomes to some extent. To determine whether similar indicators could be identified in PSCCTh, we performed a further analysis of the prognostic factors (mainly focused on treatment) after stratifying patients by age, EOD status, and clinical stage (as shown in Tables S2-S6).

As shown in Table 4, N0 stage [OS, HR: 1.887, 95\% CI: (0.939-3.574), $\mathrm{P}=0.014$; DSS, HR: $2.048,95 \% \mathrm{CI}$ : (1.306-4.217), $\mathrm{P}=0.011]$ or having received chemotherapy [OS, HR: 0.449, 95\% CI: (0.262-0.915), P=0.012; DSS, HR: 0.563 , 95\% CI: $(0.218-0.905), \mathrm{P}=0.010$ ] could predict increased OS and DSS in younger PSCCTh patients 
Table 4 Multivariable Cox proportional hazards analysis for OS stratified by the subgroups of age

\begin{tabular}{|c|c|c|c|c|}
\hline Characteristics & \multicolumn{2}{|c|}{ OS (age <70) } & \multicolumn{2}{|c|}{ OS (age $\geq 70$ ) } \\
\hline \multicolumn{5}{|l|}{ Tumor size } \\
\hline$\geq 50$ vs. $<50$ & $1.875(0.751-3.629)$ & 0.124 & $1.347(0.638-2.843)$ & $0.041^{*}$ \\
\hline \multicolumn{5}{|l|}{ T classification } \\
\hline \multicolumn{5}{|l|}{$\mathrm{N}$ classification } \\
\hline N1 vs. N0 & $1.887(0.939-3.574)$ & $0.014^{*}$ & $1.177(0.633-2.287)$ & 0.074 \\
\hline \multicolumn{5}{|l|}{ Surgery treatment } \\
\hline Surgery only vs. no/unknown & $0.538(0.256-0.941)$ & $0.017^{\star}$ & $0.498(0.199-0.852)$ & $0.003^{*}$ \\
\hline Yes vs. no/unknown & $0.449(0.262-0.915)$ & $0.012^{\star}$ & $0.710(0.318-1.939)$ & 0.091 \\
\hline
\end{tabular}

*, $\mathrm{P}<0.05$. OS, overall survival; $\mathrm{HR}$, hazard ratio; $\mathrm{Cl}$, confidence interval.

(age $<70$ ) but not in older PSCCTh patients (age $\geq 70$ ). Moreover, surgery might increase OS and DSS in all patients.

In patients without EOD, surgery with radiation was found to be an independent predictor of improved OS [HR: $0.535,95 \%$ CI: $(0.378-0.756), \mathrm{P}<0.001]$ and DSS [HR: $0.702,95 \%$ CI: $(0.446-0.989), \mathrm{P}<0.001]$, and LCN squamous cell carcinoma was the best predictor for OS [HR: 2.024, 95\% CI: (0.977-4.193), $\mathrm{P}=0.004]$ and DSS [HR: 2.988, 95\% CI: (1.788-6.017), $\mathrm{P}=0.007]$, with a median survival of 30.5 months. Age was the only independent prognostic factor in patients with EOD [OS, HR: 6.130, 95\% CI: (1.711-21.964), $\mathrm{P}=0.005$; DSS, HR: 5.234, 95\% CI: (1.096-17.407), $\mathrm{P}=0.003]$.

For advanced-stage patients, regardless of age, T stage, or $\mathrm{N}$ stage, undergoing surgery with radiation could improve their OS and DSS to a greater degree than undergoing surgery alone, while there was no significant difference between these factors for early-stage patients, suggesting that radiotherapy might be an important factor for improving the prognosis of advanced-stage patients.

\section{Establishment and validation of the nomogram model for predicting prognosis}

To produce accurate and visual outcome predictions, we constructed a nomogram model for this cohort of PSCCTh patients using the independent prognostic factors in the above multivariate Cox analyses.

The probability of 1-, 3-, and 5-year OS in PSCCTh patients was predicted in the nomogram model using total scores based on the cumulative scaled values for age, $\mathrm{T}$ stage, $\mathrm{N}$ stage, and treatment (Figure 3). We analyzed the performance of our nomogram model by comparing the consistency and discrimination between the predicted and actual events for PSCCTh patients using calibration, ROC, and C-index assays. As shown in Figure 4, the calibration curves (bootstrapping, $n=1,000$ ) showed a good fit for the predicted survival probability with the actual survival probability for the 1-, 3-, and 5-year time points. The C-index of the nomogram model was 0.871 , and the AUC results of the 1-, 3-, and 5-year OS were 0.937 (95\% CI: 0.918-0.966), 0.894 (95\% CI: 0.872-0.911), and 0.928 (95\% CI: 0.905-0.952), respectively (Figure 5), proving that the model had a good discriminatory capacity and potential for clinical application.

To test the advantages of our nomogram model, we compared it to the TNM staging model. The C-index of the TNM model was 0.658. The AUC results of TNM staging for 1-, 3-, and 5-year OS were 0.664 (95\% CI: $0.551-0.778), 0.662$ (95\% CI: $0.465-0.858)$, and 0.648 (95\% CI: $0.413-0.883)$, respectively, indicating that the utility of TNM staging for predicting PSCCTh prognosis is much lower than that of our nomogram model. 


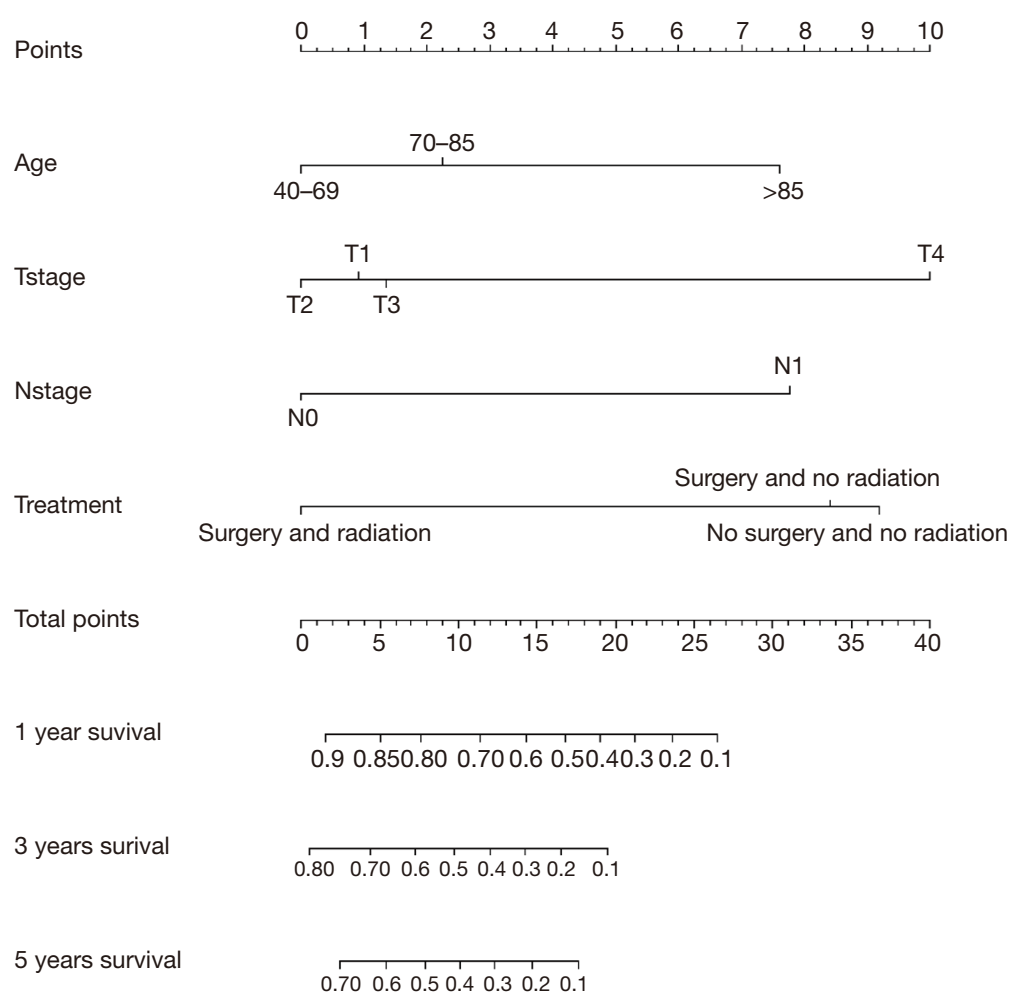

Figure 3 Nomogram for predicting 1-, 3-, and 5-year OS of PSCCTh cases. For each patient, four lines are drawn upward to determine the points received from the four predictors in the nomogram. The sum of these points is located on the "total points" axis. In addition, a line is drawn downward to determine the possibility of 1-, 3-, and 5-year OS. OS, overall survival; PSCCTh, primary squamous cell carcinoma of the thyroid.

\section{Discussion}

PSCCTh is a rare malignancy accounting for approximately $1 \%$ of thyroid cancers, making it a challenge to investigate in depth due to the limited number of cases at a single institution. As opposed to previous case reports or small cohort studies, we applied the U.S. nation-wide database named "SEER" to overcome the bias due to the small sample size. Here, we showed that PSCCTh was slightly more common in females than males, consistent with published case reports and reviews (29,30). This finding may assist head and neck physicians in predicting the location of the primary lesion if a patient has laryngeal and thyroid squamous cell carcinoma simultaneously, as it is well known that the incidence of laryngeal cancer is six times higher in males than females.

In this PSCCTh study, similar to papillary thyroid cancer, age was found to be an important metric for predicting survival time and confounding TNM stages. PSCCTh patients less than 70 years of age may have better survival than older patients regardless of other clinical parameters. The prognosis was related to tumor grade and undergoing chemotherapy in the younger patient group ( $<70$ years) but not in the older group ( $\geq 70$ years). If this finding is confirmed in a larger number of future studies, then 70 years may be a potential age threshold to be incorporated in the TNM stage system, similar to the impact of age over 55 years in papillary thyroid cancer according to the $2020 \mathrm{NCCN}$ guideline (https://www.nccn. org). This finding could assist clinicians in determining whether patients need radiotherapy.

In practice, TNM stage has been a universal factor for indirectly describing patient outcomes since 1953 . However, the pitfalls surrounding predicting prognosis based on the TNM stage are obvious. For instance, without incorporating other independent prognostic variables, such as age, gender, treatment, genetic differences, or histology, it is difficult to correlate prognosis with the limited descriptive variables of the TNM stage to accurately 

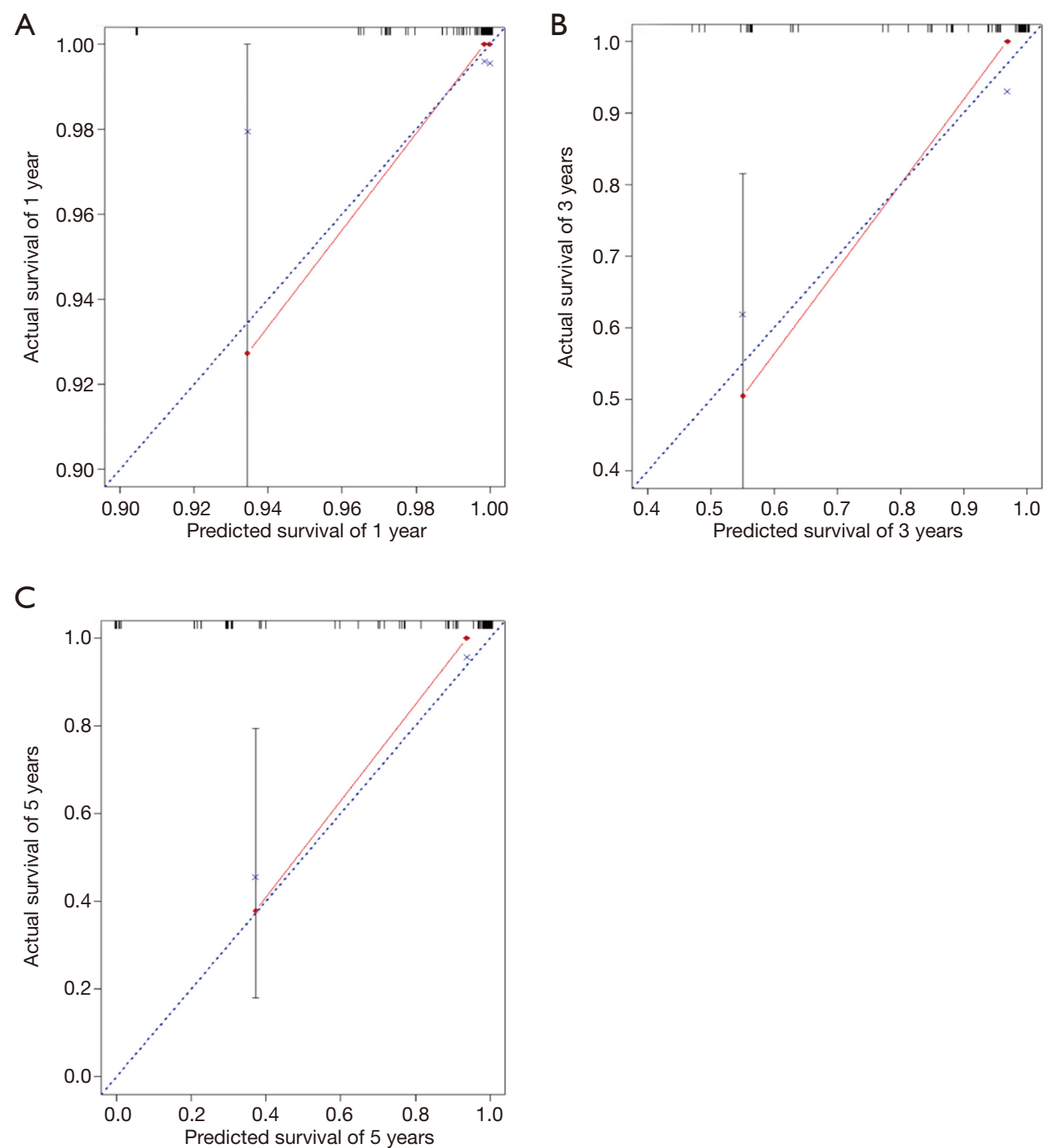

Figure 4 The calibration plots for predicting the probability of 1-year (A), 3-year (B) and 5-year (C) in the training set for OS by bootstrapping $(\mathrm{n}=1,000)$. OS, overall survival.

predict patient outcomes. To overcome the limitations of TNM staging, the nomogram model can incorporate any categorized and continuous variables relevant to prognosis and offer more user-friendly and quantitated scores for interpreting the predicted survival. To better understand the prognosis of PSCCTh, we constructed a nomogram model with four variables, including age, $\mathrm{T}$ stage, $\mathrm{N}$ stage, and surgical treatment. To the best of our knowledge, this is the first nomogram model established for PSCCTh. Furthermore, the ability of our model to distinguish varying prognoses in PSCCTh patients was confirmed to be superior to the TNM system via multiple assays, such as the C-index and ROC analysis. If the model we constructed could be further validated in an additional dataset in the future, then it would be of great assistance in physician decision-making and counseling.

Despite these findings and the innovations mentioned above in this study, we should be aware of some limitations that may confound the conclusion to some extent. The nomogram model typically needs to be validated by external data. Unfortunately, there is no additional PSCCTh dataset available for us to use to perform the external validation. Therefore, we had no choice but to employ internal bootstrapping validation as an alternative option. 


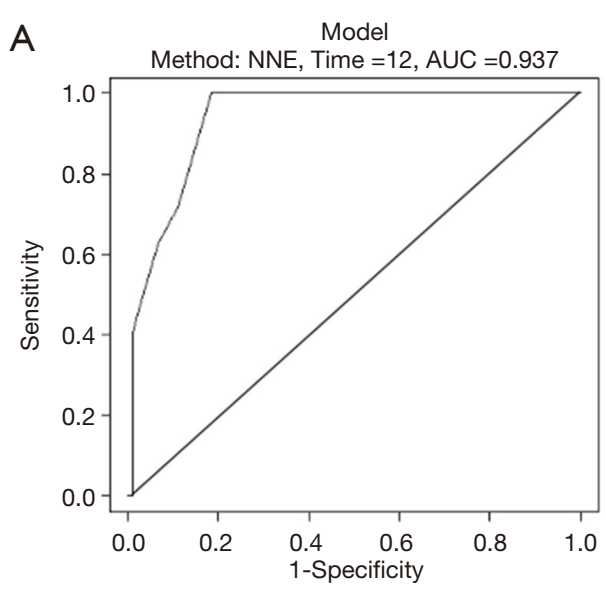

AUC at 1-year OS 0.937 (95\% Cl: 0.918-0.966)

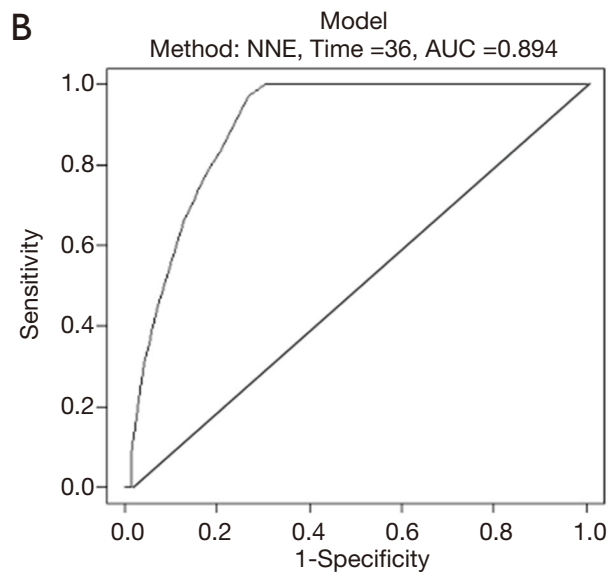

AUC at 3-year OS 0.894 (95\% Cl: 0.872-0.911)

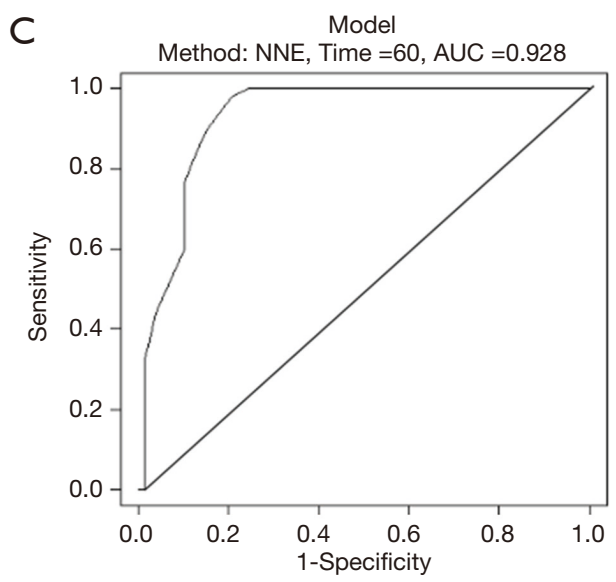

AUC at 5-year OS 0.928 (95\% Cl: 0.905-0.952)

Figure 5 ROC curves of the nomogram in prediction of prognosis at 1-year (A), 3-year (B), and 5-year point (C). ROC, receiver operating characteristic curve; AUC, area under the ROC curve; OS, overall survival; CI, confidence interval.

Additionally, there was unknown or missing information in this dataset, which could introduce bias. To reduce errors caused by missing data as much as possible, we used SPSS software to analyze the missing data, get the abstract of the missing data and ten missing modes, and identify the data type was missing at random (MAR). Then we used a mean completer for continuous variables and multiple imputations for categorical variables to evaluate the MAR data. However, regardless of the filling method used, the influence of subjective factors on the original system cannot be eliminated. Besides, we need to point out that the highly heterogeneous treatment modality might make some biases in the conclusion. The best way to eliminate these biases is the control variables. However, in actual clinical practice, it is almost difficult to have such control variables. And more details of these data cannot be identified in the SEER database. In future research, we will strive to collect more available information, select appropriate data for comparative analysis, and thereby reduce the impact of heterogeneity.

\section{Conclusions}

PSCCTh is a rare malignant tumor with high invasiveness and poor prognosis. This study showed that factors such as patient age, $\mathrm{T}$ stage, $\mathrm{N}$ stage, and surgical treatment are independent predictors of OS. PSCCTh patients who receive an early diagnosis and complete surgery 
with adjuvant radiation may have a better prognosis. The nomograms for PSCCTh prognosis predictions could help clinicians predict prognosis more accurately and provide individualized treatment.

\section{Acknowledgments}

The authors would like to thank Central South University, China, for providing necessary assistance for this study. The authors also wish to thank the Otolaryngology Major Disease Research Key Laboratory of Hunan Province, China for their support.

Funding: This work was supported by the Project of Hunan Health Commission (B2019165), National Natural Science Foundation of China (No. 81974424, 81874133, 81772903, and 81602389), Natural Science Foundation of Hunan Province (No. 2020JJ4827, 2019JJ50944, and 2018JJ2630) and the Huxiang Young Talent Project (No. 2018RS3024).

\section{Footnote}

Reporting Checklist: The authors have completed the TRIPOD reporting checklist. Available at http://dx.doi. org/10.21037/gs-20-847

Conflicts of Interest: All authors have completed the ICMJE uniform disclosure form (available at http://dx.doi. org/10.21037/gs-20-847). The authors have no conflicts of interest to declare.

Ethical Statement: The authors are accountable for all aspects of the work in ensuring that questions related to the accuracy or integrity of any part of the work are appropriately investigated and resolved. The study was conducted in accordance with the Declaration of Helsinki (as revised in 2013). Because the data in the SEER database contain no personal identifiers and are publicly available after permission is obtained, the Medical Ethics Committee of Xiangya Hospital, Central South University in Changsha, China exempted this study from review.

Open Access Statement: This is an Open Access article distributed in accordance with the Creative Commons Attribution-NonCommercial-NoDerivs 4.0 International License (CC BY-NC-ND 4.0), which permits the noncommercial replication and distribution of the article with the strict proviso that no changes or edits are made and the original work is properly cited (including links to both the formal publication through the relevant DOI and the license). See: https://creativecommons.org/licenses/by-nc$\mathrm{nd} / 4.0 \%$.

\section{References}

1. Syed MI, Stewart M, Syed S, et al. Squamous cell carcinoma of the thyroid gland: primary or secondary disease? J Laryngol Otol 2011;125:3-9.

2. Simpson WJ, Carruthers J. Squamous cell carcinoma of the thyroid gland. Am J Surg 1988;156:44-6.

3. Bolfi F, Domingues MA, Sobrinho-Simoes M, et al. Primary squamous cell carcinoma of the thyroid diagnosed as anaplastic carcinoma: failure in fine-needle aspiration cytology? Case Rep Pathol 2014;2014:301780.

4. Panchbhai AS. Primary squamous cell carcinoma of salivary gland: report of a rare case. J Cancer Res Ther 2015;11:664.

5. Kondo T, Ezzat S, Asa SL. Pathogenetic mechanisms in thyroid follicular-cell neoplasia. Nat Rev Cancer 2006;6:292-306.

6. Kwak HY, Chae BJ, Eom YH, et al. Does papillary thyroid carcinoma have a better prognosis with or without Hashimoto thyroiditis? Int J Clin Oncol 2015;20:463-73.

7. Jonklaas J, Sarlis NJ, Litofsky D, et al. Outcomes of patients with differentiated thyroid carcinoma following initial therapy. Thyroid 2006;16:1229-42.

8. Eustatia-Rutten CF, Corssmit EP, Biermasz NR, et al. Survival and death causes in differentiated thyroid carcinoma. J Clin Endocrinol Metab 2006;91:313-9.

9. Molinaro E, Romei C, Biagini A, et al. Anaplastic thyroid carcinoma: from clinicopathology to genetics and advanced therapies. Nat Rev Endocrinol 2017;13:644-60.

10. Smallridge RC, Copland JA. Anaplastic thyroid carcinoma: pathogenesis and emerging therapies. Clin Oncol (R Coll Radiol) 2010;22:486-97.

11. Haddad RI, Lydiatt WM, Ball DW, et al. Anaplastic thyroid carcinoma, version 2.2015. J Natl Compr Canc Netw 2015;13:1140-50.

12. De Cesare A, Di Cristofano C, Di Filippo AR, et al. Total thyroidectomy associated to chemotherapy in primary squamous cell carcinoma of the thyroid. Clin Ter 2019;170:e231-4.

13. Yasumatsu R, Sato M, Uchi R, et al. The treatment and outcome analysis of primary squamous cell carcinoma of the thyroid. Auris Nasus Larynx 2018;45:553-7.

14. Rosa M, Toronczyk K. Fine needle aspiration biopsy of three cases of squamous cell carcinoma presenting 
as a thyroid mass: cytological findings and differential diagnosis. Cytopathology 2012;23:45-9.

15. Lenzi R, Marchetti M, Muscatello L. Incidental nodal metastasis of differentiated thyroid carcinoma in neck dissection specimens from head and neck cancer patients. J Laryngol Otol 2017;131:368-371.

16. Goldberg HM, Harvey P. Squamous-cell cysts of the thyroid with special reference to the aetiology of squamous epithelium in the human thyroid. Br J Surg 1956;43:565-9.

17. Sanchez-Sosa S, Rios-Luna NP, Tamayo BR, et al. Primary squamous cell carcinoma of the thyroid arising in Hashimoto's thyroiditis in an adolescent. Pediatr Dev Pathol 2006;9:496-500.

18. Makay O, Kaya T, Ertan Y, et al. Primary squamous cell carcinoma of the thyroid: report of three cases. Endocr J 2008;55:359-64.

19. Zhang YX, Zhang B, Wu YH, et al. Primary squamous cell carcinoma of the thyroid: retrospective analysis of 28 cases. Zhonghua Er Bi Yan Hou Tou Jing Wai Ke Za Zhi 2013;48:143-7.

20. Tunio MA, Al AM, Fagih M, et al. Primary squamous cell carcinoma of thyroid: a case report and review of literature. Head Neck Oncol 2012;4:8.

21. Peng KA, Grogan T, Wang MB. Head and neck sarcomas: analysis of the SEER database. Otolaryngol Head Neck Surg 2014;151:627-33.

22. Au JK, Alonso J, Kuan EC, et al. Primary squamous cell carcinoma of the thyroid: a population-based analysis. Otolaryngol Head Neck Surg 2017;157:25-9.

Cite this article as: Zhang $\mathrm{X}$, Zhu G, Tang B, Huang $\mathrm{H}$, Chen C, Zheng S, Pu Y, Xu Y, Wang G, Huang D, Liu Y, Zhang $\mathrm{X}$. A characterization and prognosis prediction model for primary squamous cell carcinoma of the thyroid. Gland Surg 2021;10(4):1325-1338. doi: 10.21037/gs-20-847
23. Yang S, Li C, Shi X, et al. Primary squamous cell carcinoma in the thyroid gland: a population-based analysis using the SEER database. World J Surg 2019;43:1249-55.

24. Limberg J, Ullmann TM, Stefanova D, et al. Prognostic characteristics of primary squamous cell carcinoma of the thyroid: a national cancer database analysis. World J Surg 2020;44:348-55.

25. Fujinami K, Kondo I, Miura T, et al. Prognostic significance of clinical stage including extent of disease (EOD) in prostate cancer. Hinyokika Kiyo 1998;44:25963.

26. Vrieze SI. Model selection and psychological theory: a discussion of the differences between the Akaike information criterion (AIC) and the Bayesian information criterion (BIC). Psychol Methods 2012;17:228-43.

27. Tang LQ, Li CF, Li J, et al. Establishment and validation of prognostic nomograms for endemic nasopharyngeal carcinoma. J Natl Cancer Inst 2015;108:djv291.

28. Iasonos A, Schrag D, Raj GV, et al. How to build and interpret a nomogram for cancer prognosis. J Clin Oncol 2008;26:1364-70.

29. Booya F, Sebo TJ, Kasperbauer JL, et al. Primary squamous cell carcinoma of the thyroid: report of ten cases. Thyroid 2006;16:89-93.

30. Struller F, Senne M, Falch C, et al. Primary squamous cell carcinoma of the thyroid: Case report and systematic review of the literature. Int J Surg Case Rep 2017;37:36-40. 


\section{Supplementary}

Table S1 Comparative tests of prognostic factors for primary squamous cell carcinoma in thyroid

\begin{tabular}{lccccccccccc}
\hline Characteristics & Gender & Size & Age & T stage & N stage & M stage & Grade & Surgery & Subtype & Chemotherapy \\
\hline Gender & - & 0.290 & 0.915 & 0.175 & 0.341 & 0.587 & 0.776 & 0.156 & 0.322 & 0.800 \\
Size & 0.290 & - & 0.882 & 0.228 & 0.290 & 0.672 & 0.552 & 0.646 & 0.094 & 0.530 \\
Age & 0.915 & 0.882 & - & 0.370 & 0.915 & 0.751 & $0.013^{*}$ & 0.086 & 0.132 & 0.882 \\
T stage & 0.175 & 0.228 & 0.370 & - & 0.704 & 0.672 & 0.065 & 0.129 & 0.244 & 0.426 \\
N stage & 0.341 & 0.290 & 0.915 & 0.704 & - & $0.036^{*}$ & 0.247 & 0.760 & 0.830 & 0.056 \\
M stage & 0.587 & 0.672 & 0.751 & 0.672 & 0.036 & - & 0.160 & $0.045^{\star}$ & 0.353 & 0.184 \\
Grade & 0.776 & 0.552 & $0.013^{\star}$ & 0.065 & 0.247 & 0.160 & - & 0.160 & 0.059 & 0.270 \\
Surgery & 0.156 & 0.646 & 0.086 & 0.129 & 0.760 & $0.045^{\star}$ & 0.160 & - & 0.353 & $0.021^{*}$ \\
Subtype & 0.322 & 0.094 & 0.132 & 0.244 & 0.830 & 0.353 & 0.059 & 0.353 & - & 0.442 \\
Chemotherapy & 0.800 & 0.530 & 0.882 & 0.426 & 0.056 & 0.184 & 0.270 & $0.021^{*}$ & 0.442 & - \\
\hline
\end{tabular}

*, $\mathrm{P}<0.05$.

Table S2 Univariate Cox proportional hazards analysis stratified by the subgroups of age

\begin{tabular}{|c|c|c|c|c|}
\hline Characteristics & \multicolumn{4}{|c|}{ Univariate log-rank $P$ value } \\
\hline \multicolumn{5}{|l|}{ Gender } \\
\hline Female vs. male & 0.537 & 0.754 & 0.896 & 0.223 \\
\hline \multicolumn{5}{|l|}{ Tumor size } \\
\hline$\geq 50$ vs. $<50$ & 0.212 & $0.021^{*}$ & 0.052 & $0.019^{*}$ \\
\hline \multicolumn{5}{|l|}{ EOD } \\
\hline Yes vs. no/unknown & 0.298 & $0.027^{*}$ & 0.627 & 0.464 \\
\hline \multicolumn{5}{|l|}{ T classification } \\
\hline T4 vs. T1-3 & $0.005^{*}$ & $0.006^{*}$ & $0.005^{\star}$ & $0.004^{*}$ \\
\hline \multicolumn{5}{|l|}{$\mathrm{N}$ classification } \\
\hline M1 vs. M0 & $0.034^{*}$ & 0.165 & $0.029^{*}$ & 0.782 \\
\hline \multicolumn{5}{|l|}{ Grade } \\
\hline Grade III-IV vs. I-II & $<0.001^{*}$ & 0.995 & $0.001^{*}$ & 0.951 \\
\hline \multicolumn{5}{|l|}{ Subtype } \\
\hline Keratinizing/LCN/SC/MI vs. NOS & 0.443 & 0.163 & 0.632 & 0.848 \\
\hline \multicolumn{5}{|l|}{ Surgery treatment } \\
\hline Surgery only vs. no/unknown & $0.002^{*}$ & $0.001^{*}$ & $0.015^{\star}$ & $0.012^{*}$ \\
\hline Surgery + radiation vs. no/unknown & $0.011^{*}$ & $<0.001^{*}$ & $0.031^{*}$ & $0.010^{\star}$ \\
\hline
\end{tabular}

${ }^{*}, \mathrm{P}<0.05$. OS, overall survival; DSS, disease-specific survival; EOD, extent of disease; LCN, large cell, nonkeratinizing; SC, spindle cell; MI, micro-invasive; NOS, not otherwise specified. 
Table S3 Multivariable Cox proportional hazards analysis for DSS stratified by the subgroups of age

\begin{tabular}{|c|c|c|c|c|}
\hline Characteristics & \multicolumn{2}{|c|}{ DSS (age <70) } & \multicolumn{2}{|c|}{ DSS (age $\geq 70$ ) } \\
\hline \multicolumn{5}{|l|}{ Tumor size } \\
\hline$\geq 50$ vs. $<50$ & $1.244(0.385-2.711)$ & 0.088 & $1.958(1.104-5.325)$ & $0.049^{*}$ \\
\hline \multicolumn{5}{|l|}{ T classification } \\
\hline \multicolumn{5}{|l|}{$\mathrm{N}$ classification } \\
\hline N1 vs. N0 & $2.048(1.306-4.217)$ & $0.011^{*}$ & $1.021(0.403-2.228)$ & 0.066 \\
\hline \multicolumn{5}{|l|}{ Surgery treatment } \\
\hline Surgery only vs. no/unknown & $0.474(0.141-0.623)$ & $0.006^{\star}$ & $0.504(0.177-0.993)$ & $0.001^{*}$ \\
\hline Yes vs. no/unknown & $0.563(0.218-0.905)$ & $0.010^{\star}$ & $0.520(0.298-1.834)$ & 0.053 \\
\hline
\end{tabular}

${ }^{*}, \mathrm{P}<0.05$. DSS, disease-specific survival; $\mathrm{HR}$, hazard ratio; $\mathrm{Cl}$, confidence interval.

Table S4 Univariate Cox proportional hazards analysis stratified by the subgroups of EOD

\begin{tabular}{|c|c|c|c|c|}
\hline Characteristics & \multicolumn{4}{|c|}{ Univariate log-rank $P$ value } \\
\hline \multicolumn{5}{|l|}{ Gender } \\
\hline Female vs. male & 0.965 & 0.884 & 0.965 & 0.685 \\
\hline \multicolumn{5}{|l|}{ Age (years old) } \\
\hline \multicolumn{5}{|l|}{ EOD } \\
\hline Yes vs. no/unknown & 0.640 & 0.892 & 0.640 & 0.055 \\
\hline \multicolumn{5}{|l|}{ Grade } \\
\hline Grade III-IV vs. I-II & 0.531 & 0.979 & 0.531 & 0.586 \\
\hline \multicolumn{5}{|l|}{ Surgery treatment } \\
\hline Surgery only vs. no/unknown & 0.754 & $0.034^{*}$ & 0.754 & $0.011^{\star}$ \\
\hline Surgery + radiation vs. no/unknown & 0.872 & $0.045^{\star}$ & 0.872 & $0.016^{\star}$ \\
\hline \multicolumn{5}{|l|}{ Chemotherapy } \\
\hline Yes vs. no/unknown & 0.793 & 0.756 & 0.793 & 0.212 \\
\hline
\end{tabular}

*, P<0.05. EOD, extent of disease; OS, overall survival; DSS, disease-specific survival; LCN, large cell, nonkeratinizing; SC, spindle cell; $\mathrm{MI}$, micro-invasive; NOS, not otherwise specified. 
Table S5 Multivariable Cox proportional hazards analysis stratified by the subgroups of no EOD

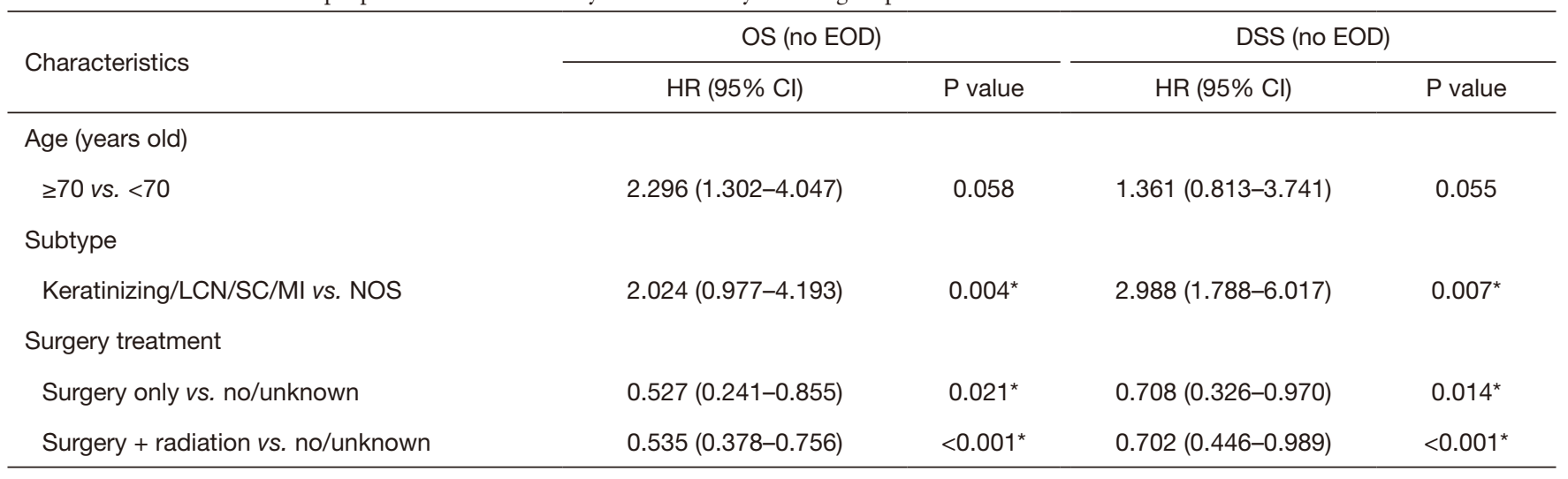

* $\mathrm{P}<0.05$. EOD, extent of disease; OS, overall survival; DSS, disease-specific survival; HR, hazard ratio; Cl, confidence interval; LCN, large cell, nonkeratinizing; SC, spindle cell; MI, micro-invasive; NOS, not otherwise specified.

Table S6 Kaplan-Meier survival analysis stratified by the subgroups of surgery treatment

\begin{tabular}{|c|c|c|c|c|c|c|}
\hline Characteristics & \multicolumn{6}{|c|}{ Univariate log-rank $P$ value } \\
\hline \multicolumn{7}{|l|}{ EOD } \\
\hline No & $0.003^{*}$ & $0.002^{*}$ & 0.424 & 0.527 & 0.107 & 0.095 \\
\hline Yes & 0.244 & 0.417 & 0.464 & 0.384 & 0.730 & 0.813 \\
\hline \multicolumn{7}{|l|}{ Age } \\
\hline$<70$ years old & $0.024^{*}$ & $0.023^{*}$ & 0.087 & 0.071 & $0.002^{*}$ & $0.001^{*}$ \\
\hline$\geq 70$ years old & $<0.001^{*}$ & $<0.001^{*}$ & $0.008^{\star}$ & $0.005^{\star}$ & $0.035^{\star}$ & $0.004^{*}$ \\
\hline \multicolumn{7}{|l|}{ T classification } \\
\hline $\mathrm{T} 1-\mathrm{T} 3$ & $0.001^{*}$ & $0.001^{*}$ & 0.497 & 0.502 & $0.016^{*}$ & $0.012^{*}$ \\
\hline \multicolumn{7}{|l|}{$\mathrm{N}$ classification } \\
\hline No & $0.017^{\star}$ & $0.008^{*}$ & 0.148 & 0.052 & 0.193 & 0.202 \\
\hline N1 & $0.001^{*}$ & $0.001^{*}$ & $0.022^{*}$ & $0.012^{\star}$ & 0.951 & 0.872 \\
\hline
\end{tabular}

*, P<0.05. OS, overall survival; DSS, disease-specific survival; EOD, extent of disease; SR, surgery with radiation; SO, surgery only; NS, no surgery. 